\title{
ESTUDIO EXPERIMENTAL Y NUMÉRICO PARA DETERMINAR OPORTUNIDADES DE AHORRO DE ENERGÍA Y REDUCCIÓN DE LA TEMPERATURA AMBIENTE EN UNA VIVIENDA ECONÓMICA TÍPICA EN LA CIUDAD DE LA PAZ, B.C.S. MÉXICO
}

\section{EXPERIMENTAL AND NUMERICAL STUDY TO DETERMINE OPPORTUNITIES FOR ENERGY SAVING AND REDUCTION OF THE AMBIENT TEMPERATURE IN A TYPICAL ECONOMIC HOME IN THE CITY OF LA PAZ, B.C.S. MEXICO}

\section{GALINDO DE LA CRUZ, Madelein}

Departamento Académico de Ingeniería en Pesquerías Universidad Autónoma de Baja California Sur (UABCS)

Carretera al sur km 5.5, La Paz, Baja California Sur. C.P. 23080. México

E-mail: mgalindo@uabcs.mx

Teléfono: +52 (612) 1238800 extensión 6210 y 4614

\section{RESÉNDIZ PACHECO, Oscar}

Departamento Académico de Ingeniería en Pesquerías Universidad Autónoma de Baja California Sur (UABCS)

Carretera al sur km 5.5, La Paz, Baja California Sur. C.P. 23080. México

E-mail: resendiz@uabcs.mx

Teléfono: +52 (612) 1238800 extensión 6210 y 4614

\section{LUNA LEÓN, Aníbal}

Profesor investigador. Posgrado e Investigación, Facultad de Arquitectura y Diseño Universidad Autónoma de Baja California (UABC)

Calle de la Normal e Ignacio López Rayón S/N, 3er Piso, Colonia Insurgentes Este. Unidad Mexicali, Mexicali, B.C., C.P. 21900, México

E-mail. ramonaaliciaromero@yahoo.com.mx

Teléfono: +52 (686) 5-66-42-50.

\section{POUJOL, Federico}

Departamento Académico de Ingeniería en Pesquerías

Universidad Autónoma de Baja California Sur (UABCS)

Carretera al sur km 5.5, La Paz, Baja California Sur. C.P. 23080. México

E-mail: ftpoujol@uabcs.mx

Teléfono: +52 (612) 1238800 extensión 6210 y 4614

Palabras Clave: Ahorro de energía; estrategias pasivas; temperatura del aire; aislamiento térmico

Keywords: Energy saving; passive strategies; air temperature; thermal insulation 


\section{Resumen}

El presente trabajo se elaboró llevando a cabo un estudio con el fin de identificar estrategias pasivas de reducción de la temperatura interior y ahorro de energía en una casa habitación de interés social en la Paz B.C.S. Para dicho estudio se utilizó una vivienda ubicada en la colonia Calafia en esta ciudad a la cual se le aplicaron diferentes espesores de un material de recubrimiento a base de celulosa. A dicha vivienda se le determinó la temperatura interior del aire, en diferentes ubicaciones, así como las temperaturas de las paredes, piso y techo. Se determinaron también la temperatura exterior del aire y se obtuvo de una estación meteorológica de la CNA la radiación en los días que se llevaron a cabo las pruebas. Con los datos experimentales se determinó la aminoración de la temperatura ambiente. Estos resultados se compararon con los de simulaciones de una casa de geometría similar con diversas estrategias de aislamiento. Se concluye que se puede amortiguar más la temperatura interior de la casa medida aumentando el espesor y la masa de los materiales aislantes, apoyado con el uso de elementos de sombreado y vegetación.

\section{Abstract}

The present work was elaborated carrying out a study with the purpose of identifying passive strategies of reduction of the interior temperature and energy saving in a house of social interest in La Paz B.C.S. For this study, a house located in the Calafia neighborhood was used in this city to which different thicknesses of a cellulose-based coating material were applied. The interior temperature of the air, in different locations as well as the temperatures of the walls, floor and ceiling, was determined to said dwelling. The external temperature of the air was also determined and the radiation was obtained from a CNA meteorological station on the days that the tests were carried out. With the experimental data, the reduction of the ambient temperature was determined. These results were compared with simulations of a house of similar geometry with different isolation strategies. It is concluded that the interior temperature of the house can be further dampened by increasing the thickness and mass of the insulating materials, supported by the use of shading and vegetation elements.

\section{Introducción}

Uno de los problemas más importantes relacionados con el ahorro de energía tiene que ver con el consumo de electricidad para el acondicionamiento de aire en industrias, casas habitación, oficinas, escuelas y en todas las edificaciones donde exista concentración de personas.

Es por lo anterior que se han elaborado propuestas muy variadas en las que se coincide que una estrategia importante para atacar este problema comprende la aplicación de mejores y más eficientes materiales de recubrimiento térmico aplicados en las construcciones.

Recientemente se han propuesto como aislamiento térmico materiales a base de celulosa debido a su bajo costo de producción, sus características físicas y a que la materia prima que los conforma es simplemente papel reciclado y elementos adhesivos.

La tarea de comprobar si realmente esta nueva generación de materiales térmicos funciona, se puede apoyar en tecnologías computacionales que permiten simular el comportamiento térmico

Citación: GALINDO DE LA CRUZ, M. et al. Estudio experimental y numérico para determinar oportunidades de ahorro de energía y reducción de la temperatura ambiente en una vivienda económica típica en la ciudad de La Paz, B.C.S. México. En: Libro de proceedings, CTV 2018. XII Congreso Internacional Ciudad y Territorio Virtual. "Ciudades y Territorios Inteligentes". UNCuyo, Mendoza, 5-7 septiembre 2018. Barcelona: CPSV, 2018, p. 331-338. 
de un proyecto de construcción, ayudando así a la toma de decisiones de los ingenieros proyectistas respecto de la selección de materiales que tendrán los resultados esperados. Uno de los programas utilizados para este propósito es el Energy Plus, programa computacional de protocolo abierto, creado por el Departamento de Energía de Estados Unidos. Este programa es capaz de calcular la temperatura interna o la ganancia térmica de un edificio en intervalos de tiempo determinados.

\section{Metodología}

\subsection{Obtención de Datos para la Simulación}

Estudio o levantamiento de medidas del edificio: Utilizando el plano de construcción del predio y realizando levantamiento de medidas directamente del mismo, se procedió a recabar la información geométrica de la construcción, observando su orientación, y tomando nota de su material de elaboración.

Creación de imagen tridimensional: Con la ayuda del Google Sketchup se realizó el modelado tridimensional de la construcción, especificando las medidas reales de los espacios, así como grosor de muros y de materiales de recubrimiento.

\subsection{Localización del Predio}

El predio se encuentra ubicado en la ciudad de La Paz, Baja California Sur y a su vez cabecera del municipio de La Paz. Se ubica al noroeste de México. Se encuentra a 215 kilómetros al sur de Ciudad Constitución, municipio de Comondú y a 202 al norte de Cabo San Lucas, municipio de Los Cabos. Sus coordenadas geográficas son $24^{\circ} 09^{\prime}$ Norte y paralelo $110^{\circ} 19^{\prime}$ Oeste, tiene una altitud de 0 a 27 metros sobre el nivel del mar. (Cuaderno estadístico municipal de la Paz B C S 1998). Dicha construcción es una casa habitación de interés social de carácter privada. Se encuentra en la colonia Cola de Ballena, fraccionamiento de nueva creación, ubicado en carretera al sur $\mathrm{Km}$. 17. Colinda al sur-oeste con la colonia Calafia. Con coordenadas geográficas $24^{\circ} 01^{\prime} 44.4^{\prime \prime} \mathrm{N}, 110^{\circ} 16^{\prime} 03.1^{\prime \prime} \mathrm{W}$.

\subsection{Clima}

El clima de la ciudad de La Paz está influenciado por la zona geográfica inmediata a la misma. Por su latitud se encuentra en la franja donde se sitúan los mayores desiertos del mundo. También es de resaltar que la ciudad de La Paz se encuentra justo a unos cuantos kilómetros arriba del Trópico de Cáncer el cual es una línea imaginaria que señala los límites globales de las zonas tropicales con clima cálido.

\subsection{Descripción Geométrica y Constructiva de la Vivienda}

La construcción sometida a estudio es una vivienda de interés social de $44 \mathrm{~m}^{2}$ de construcción, la cual cuenta con dos recámaras, un baño, una cocina y una sala. La Figura 1 muestra la distribución geométrica y constructiva de la vivienda, así como las medidas reales de las áreas que conforman la misma.

Citación: GALINDO DE LA CRUZ, M. et al. Estudio experimental y numérico para determinar oportunidades de ahorro de energía y reducción de la temperatura ambiente en una vivienda económica típica en la ciudad de La Paz, B.C.S. México. En: Libro de proceedings, CTV 2018. XII Congreso Internacional Ciudad y Territorio Virtual. "Ciudades y Territorios Inteligentes". UNCuyo, Mendoza, 5-7 septiembre 2018. Barcelona: CPSV, 2018, p. 331-338. 
El primer plano de la figura es la fachada, la cual se considera orientada hacia el suroeste. Las recámaras se encuentran en la parte posterior. La vivienda es parte de una vivienda dúplex. Las paredes del lado izquierdo (divisorias de la casa dúplex) no se consideran asoleadas, se toman adiabáticas y sin acumulación térmica.

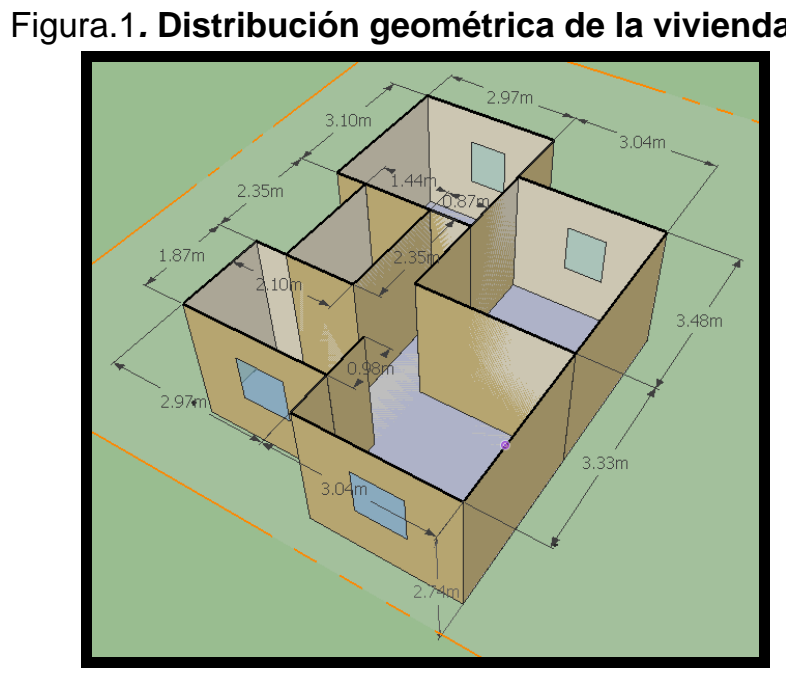

Fuente: Elaboración propia

La Tabla1 muestra las características termo-físicas y espesores de los materiales utilizados para la construcción de las paredes interiores y exteriores de la casa en estudio. En el estudio, un caso simulado (caso 1) fue el de la casa base en la que los muros exteriores constan de una capa de block de concreto y 2 capas, una interna y otra externa, de mortero. El otro caso simulado (caso 2) fue el de la casa base con paredes exteriores de la misma composición, pero con el agregado exterior de una capa de recubrimiento de celulosa de $1.56 \mathrm{~mm}$ (1/16 de pulgada).

Tabla 1. Composición y Propiedades Termo Físicas de los Muros Exteriores e Interiores

Fuente: Elaboración propia

\begin{tabular}{|l|c|c|c|}
\hline \multirow{2}{*}{$\begin{array}{c}\text { Dimensiones } y \\
\text { propiedades termo } \\
\text { físicas }\end{array}$} & $\begin{array}{c}|c| \\
\text { Recubrimiento } \\
\text { de Celulosa }\end{array}$ & $\begin{array}{c}\text { Block } \\
\text { concreto }\end{array}$ & $\begin{array}{c}\text { Recubrimiento } \\
\text { Mortero-Arena }\end{array}$ \\
\cline { 2 - 4 } & 1.56 & 150 & 2 \\
\hline Espesor $(\mathrm{mm})$ & 45 & 1800 & 200 \\
\hline Densidad $\left(\mathrm{Kg} / \mathrm{m}^{3}\right)$ & 0.038 & 0.66 & 0.63 \\
\hline $\begin{array}{c}\text { Conductividad } \\
\text { térmica }(\mathrm{W} / \mathrm{m}-\mathrm{K})\end{array}$ & 2100 & 1000 & 873 \\
\hline $\begin{array}{l}\text { Calor } \\
\text { especifico }(\mathrm{J} / \mathrm{Kg}-\mathrm{K})\end{array}$ & Rugoso & Rugoso & Rugoso \\
\hline \multicolumn{1}{|c|}{ Rugosidad } & & & \\
\hline
\end{tabular}

La Tabla 2 muestra las características termo-físicas y espesores de los materiales utilizados para la construcción de la loza de la casa en estudio. En cuanto al techo, el caso 1 simulado incorporaba en el techo de afuera hacia adentro capas de cemento, poliestireno y yeso de los espesores indicados en dicha Tabla. El caso 2 incorporaba adicionalmente en el exterior una capa de recubrimiento de celulosa del espesor indicado en la tabla mencionada.

Citación: GALINDO DE LA CRUZ, M. et al. Estudio experimental y numérico para determinar oportunidades de ahorro de energía y reducción de la temperatura ambiente en una vivienda económica típica en la ciudad de La Paz, B.C.S. México. En: Libro de proceedings, CTV 2018. XII Congreso Internacional Ciudad y Territorio Virtual. "Ciudades y Territorios Inteligentes". UNCuyo, Mendoza, 5-7 septiembre 2018. Barcelona: CPSV, 2018, p. 331-338. 
Tabla 2. Composición y Propiedades de la Loza

\begin{tabular}{|c|c|c|c|c|}
\hline \multirow{2}{*}{$\begin{array}{c}\text { Dimensiones } y \\
\text { propiedades termo } \\
\text { físicas }\end{array}$} & $\begin{array}{c}|c| \\
\text { Recubrimiento de } \\
\text { Celulosa }\end{array}$ & $\begin{array}{c}\text { Recubrimiento } \\
\text { Cemento }\end{array}$ & Poliestireno & Yeso \\
\cline { 2 - 5 } & 1.56 & 50 & 100 & 1.9 \\
\hline Espesor (mm) & 45 & 1280 & 15 & 800 \\
\hline Densidad (Kg/m $\left.{ }^{3}\right)$ & 0.038 & 0.53 & 0.04 & 0.16 \\
\hline $\begin{array}{c}\text { Conductividad térmica } \\
(\mathrm{W} / \mathrm{m}-\mathrm{K})\end{array}$ & 2100 & 840 & 1400 & 1090 \\
\hline $\begin{array}{c}\text { Calor especifico } \\
(\mathrm{J} / \mathrm{Kg}-\mathrm{K})\end{array}$ & Rugoso & Rugoso & Media & No \\
\hline Rugosidad & & & & \\
\hline
\end{tabular}

Fuente: Elaboración propia

La Tabla 3 muestra las características termo-físicas y espesores de los materiales utilizados para la construcción del piso y cimentación la casa en estudio.

\section{Tabla 3. Composición y Propiedades de Piso y Cimentación}

\begin{tabular}{|l|c|}
\hline $\begin{array}{c}\text { Dimensiones y propiedades } \\
\text { termo físicas }\end{array}$ & $\begin{array}{c}\text { Loza Maciza d=2500 } \\
\mathrm{Kg} / \mathrm{cm}^{2}\end{array}$ \\
\hline Espesor $(\mathrm{mm})$ & 100 \\
\hline Densidad $\left(\mathrm{Kg} / \mathrm{m}^{3}\right)$ & 1280 \\
\hline Conductividad térmica $(\mathrm{W} / \mathrm{m}-\mathrm{K})$ & 0.53 \\
\hline Calor especifico $(\mathrm{J} / \mathrm{Kg}-\mathrm{K})$ & 840 \\
\hline Rugosidad & Rugoso \\
\hline
\end{tabular}

Fuente: Elaboración propia

\subsection{Modelo y Programa de Simulación}

Con el apoyo del programa Energy Plus, se llevaron a cabo simulaciones, en las cuales se especificó la aplicación de los diferentes materiales en el inmueble. La simulación se hizo para todo el año.

\section{Resultados}

\subsection{Resultados de la Simulación}

Las siguientes gráficas muestran el comportamiento de la temperatura del aire en el interior de los espacios de la construcción (recamara no adyacente, y sala-cocina-comedor), para el día 21 de julio.

La Figura 2 muestra el comportamiento de la temperatura del aire en el interior de la recámara no adyacente a la otra mitad de la casa duplex (recámara 1) un día 21 del mes de julio. Como se observa, la diferencia de temperatura que arroja la simulación entre los dos materiales de recubrimiento (sólo mortero y mortero más celulosa externa) apenas rebasa $1^{\circ} \mathrm{C}$ aproximadamente.

Citación: GALINDO DE LA CRUZ, M. et al. Estudio experimental y numérico para determinar oportunidades de ahorro de energía y reducción de la temperatura ambiente en una vivienda económica típica en la ciudad de La Paz, B.C.S. México. En: Libro de proceedings, CTV 2018. XII Congreso Internacional Ciudad y Territorio Virtual. "Ciudades y Territorios Inteligentes". UNCuyo, Mendoza, 5-7 septiembre 2018. Barcelona: CPSV, 2018, p. 331-338. 
Los dos acabados externos alcanzan una temperatura máxima aproximadamente $4^{\circ} \mathrm{C}$ menor que la máxima temperatura ambiente, la cual se presenta 4 horas antes.

\section{Figura 2. Comportamiento de la temperatura del aire en la recámara 1 en julio}

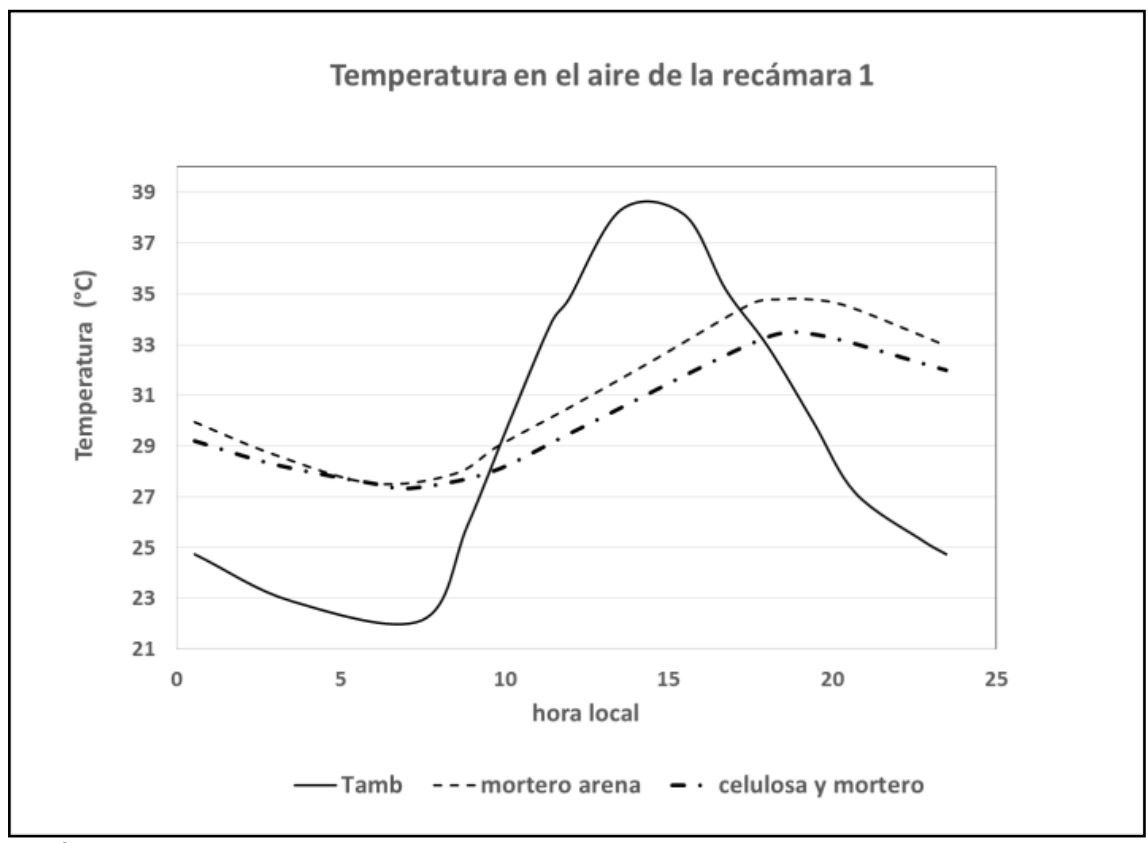

Fuente: Elaboración propia

La Figura 3 muestra el comportamiento de la temperatura en el interior del espacio que corresponde a la sala, cocina y comedor en un día típico del mes de julio en la cual se nota un comportamiento muy similar de los materiales estudiados y una diferencia de $5^{\circ} \mathrm{C}$ de sus máximos con respecto a la temperatura ambiente.

\section{Figura 3. Comportamiento de la temperatura del aire en la sala-comedor en julio}

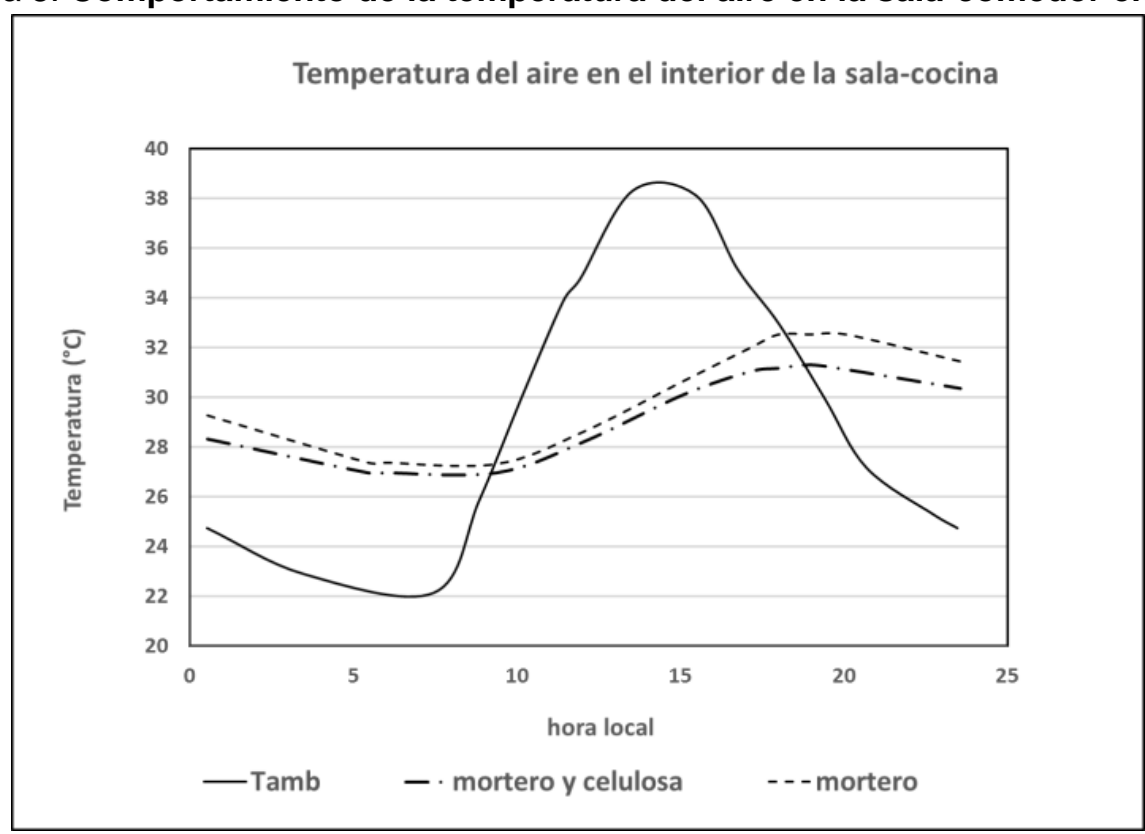

Fuente: Elaboración propia

Citación: GALINDO DE LA CRUZ, M. et al. Estudio experimental y numérico para determinar oportunidades de ahorro de energía y reducción de la temperatura ambiente en una vivienda económica típica en la ciudad de La Paz, B.C.S. México. En: Libro de proceedings, CTV 2018. XII Congreso Internacional Ciudad y Territorio Virtual. "Ciudades y Territorios Inteligentes". UNCuyo, Mendoza, 5-7 septiembre 2018. Barcelona: CPSV, 2018, p. 331-338. 


\section{Conclusiones}

Los resultados de la simulación muestran comportamientos similares para la casa recubierta con sólo mortero-arena y para la casa con recubrimiento adicional a base de celulosa.

La diferencia máxima fue de aproximadamente un grado, lo cual se esperaba en cierta forma a pesar de la gran diferencia en propiedades térmicas entre este material y los demás de las paredes ya que el espesor de recubrimiento de celulosa considerado fue pequeño $(1.56 \mathrm{~mm}$, que representa una aplicación comercial del mismo).

Si bien estas diferencias de temperatura para una casa no climatizada artificialmente no significan gran cosa en términos de confort, son indicativas del aumento de $18 \%$ en la resistencia térmica de las paredes, al aplicar aún esa pequeña capa de recubrimiento de celulosa, lo cual se traduciría en ahorros en términos de costos de energía para aire acondicionado.

Es de notar, sin embargo, que problemas de consistencia del material pueden limitar su aplicación externa en paredes verticales a pequeños espesores. Cabe mencionar que una alternativa es aplicarlo en el interior de huecos de los bloques como una manera de aumentar la resistencia térmica de la pared (DOE, 2015).

No hay que olvidar la aparentemente baja huella ecológica de este material, y que aun cuando un material se muestra superior a otro térmicamente, la decisión sobre cuál usar debe también considerar su impacto ecológico al término de la vida útil de la construcción.

\section{Bibliografía}

CADENA, C. G. y BULA SILVERA, A. J. Estudio de la variación en la conductividad térmica de la Cascarilla de arroz aglomerada con fibras vegetales. Universidad del Norte Barranquilla Colombia. 1997. 19 p.

INSTITUTO NACIONAL DE ESTADÍSTICA Y GEOGRAFÍA. Anuario Estadístico de Baja California Sur. Gobierno del Estado de Baja California Sur. 2009. 491 p.

GARCíA, J. y BOJÓRQUEZ, G. Diseño y Simulación Térmica de Vivienda Ecoturística. Facultad Arquitectura, Universidad Autónoma de Sinaloa. 2001. 6 p.

MARTIN ALCALDE, A. Estudio y simulación de un edificio con Energy Plus. Escuela técnica superior de ingeniería. 2010. 330 p.

MUÑOZ, A. y ESPINEL, J. Evolución de la celulosa de papel y de la ceniza de carbón, como materiales aislantes alternativos. Vol. 73. Universidad Nacional de Colombia. Medellín Colombia. 2006. 8 p.

OROZCO ALVARADO, C. y DUQUE, J. Simulación Térmica de un Edificio con Fines de Ahorro Energético. Escuela Superior Politécnica del Litoral. Guayaquil Colombia. 2000. 9 p.

Citación: GALINDO DE LA CRUZ, M. et al. Estudio experimental y numérico para determinar oportunidades de ahorro de energía y reducción de la temperatura ambiente en una vivienda económica típica en la ciudad de La Paz, B.C.S. México. En: Libro de proceedings, CTV 2018. XII Congreso Internacional Ciudad y Territorio Virtual. "Ciudades y Territorios Inteligentes". UNCuyo, Mendoza, 5-7 septiembre 2018. Barcelona: CPSV, 2018, p. 331-338. 
U.S. DEPARTMENT OF ENERGY'S (DOE) Types of Insulation. En: Energy Saber [En línea] Fecha de consulta: 10 de junio de 2015. Disponible en: http://www.energy.gov/energysaver/articles/types-insulation

VICTORERO CASTAÑO, F. A. y BUSTAMANTE GÓMEZ, W. Estudio del comportamiento térmico de la vivienda de emergencia o mediagua. Pontificia Universidad Católica de Chile (PUCH). Santiago de Chile, Chile. 2008. 52 p. 